\title{
PRODUÇÃO DE POLPAS DE MANGAS TOMMY ATKINS, NA AMAZÔNIA SETENTRIONAL, ATRAVÉS DA APLICAÇÃO DE PRESERVATIVOS E DA PASTEURIZAÇÃO ${ }^{1}$
}

\author{
LEANDRO CAMARGO NEVES ${ }^{2}$, RONALDO MORENO BENEDETTE ${ }^{3}$, VANUZA XAVIER DA SILVA ${ }^{4}$, \\ MARCOS ANDRÉ DE SOUZA PRILL ${ }^{4}$, ROGÉRIO LOPES VIEITES ${ }^{5}$
}

RESUMO - O presente trabalho avaliou a eficiência da pasteurização e o uso de preservativos em polpas de manga Tommy Atkins (Mangifera indica L.) refrigeradas. Antes da confecção dos tratamentos, os frutos foram higienizados em solução de hipoclorito de sódio $(\mathrm{NaOCl})$ a $100 \mathrm{mg}$. $\mathrm{L}^{-1}$, por 10 minutos. Após o processamento, foi realizado o ajuste do $\mathrm{pH}$ das polpas para 3,0, e o ajuste da atividade de água $\left(\mathrm{A}_{\mathrm{a}}\right.$ ) para 0,95 . As variáveis utilizadas foram a pasteurização (água fervente a $95 \pm 5^{\circ} \mathrm{C}$, por 0 e 1 minuto), a adição de benzoato de sódio nas concentrações de 0; 200 e $500 \mathrm{~mL}$. $\mathrm{L}^{-1}$, e dióxido de enxofre $\left(\mathrm{SO}_{2}\right)$ a $0 ; 100$ e $200 \mathrm{~mL}$. $\mathrm{L}^{-1}$. As polpas foram então embaladas em sacos de polietileno de baixa densidade (PEBD) de 0,060 mm (0,10 x 0,12 $\mathrm{m}$ - sem espaço livre e contendo 500g de polpa), e armazenadas em câmara frigorífica a $20 \pm 1{ }^{\circ} \mathrm{C} \mathrm{e} 80 \pm 3 \%$ de U.R, por 28 dias. Ao final do experimento, os tratamentos não-submetidos à pasteurização apresentaram os maiores níveis de ácido ascórbico. Entretanto, nesses mesmos tratamentos, foram intensa atividade microbiana e elevados níveis de $\mathrm{pH}$. Não foram detectadas diferenças significativas entre os tratamentos testados nas análises de acidez titulável (AT) e sólidos solúveis (SS). Devido à ausência de escurecimento nas polpas trabalhadas, durante todo o período experimental, não se podem detectar as diferenças entre a aplicação do $\mathrm{SO}_{2}$ e a utilização da pasteurização. $\mathrm{O}$ menor índice de contaminação microbiológica e a preferência dos julgadores no painel sensorial foram atribuídos aos tratamentos submetidos à pasteurização, adição de benzoato de sódio a $500 \mathrm{~mL} \cdot \mathrm{L}^{-1}$ e adição de dióxido de enxofre a $200 \mathrm{~mL} \cdot \mathrm{L}^{-1}$.

Termos para indexação: Mangifera indica L., agroindústria, propriedades físicas, análise sensorial.

\section{PRODUCTION OF MANGO PULPS TOMMY ATKINS IN SEPTENTRIONAL AMAZON THROUGH APLICATION OF PRESERVATIVES AND OF BRANCHING}

\begin{abstract}
The present work had evaluated the efficiency of the pasteurization and the use of preservatives in mangos pulps (Mangifera indica L.) refrigerated. Before carrying out the treatments, the fruits had been cleaned in solution of sodium hypochlorite $(\mathrm{NaOCl})$ at $100 \mathrm{mg}$. L ${ }^{-1}$, for 10 minutes. After the processing, it was carried out through the adjustment of $\mathrm{pH}$ of pulps for 3.0 and the adjustment of the water activity $\left(\mathrm{W}_{\mathrm{a}}\right)$ at 0.95 . The used variable had been the branching (hot water at $95 \pm 5^{\circ} \mathrm{C}$, for 0 and 1 minute), the addition of sodium benzoate in the concentrations of $0 ; 200$ and $500 \mathrm{~mL} . \mathrm{L}^{-1}$ and, sulfite dioxide $\left(\mathrm{SO}_{2}\right)$ in 0,100 and $200 \mathrm{~mL} . \mathrm{L}^{-1}$. Then the pulps had been packed in low density polyethylene (LDPE) bags of $0,060 \mathrm{~mm}(0.10 \times 0.12 \mathrm{~m}$ - without free space and content of $500 \mathrm{~g}$ of pulp) and, conditioned in at $20 \pm 1{ }^{\circ} \mathrm{C} \mathrm{e} 80 \pm 3 \%$ of R.H, for 28 days. At the end of the experiment, the treatments not submitted to the branching had shown the biggest ascorbic acid levels. However, in these same treatments had been noticed intense microbiological activity and raised levels of $\mathrm{pH}$. There aren't significant differences between the tested treatments in the titratable acidity (TA) and soluble solids (SS) analyses. Due to the absence of black spot in the pulps used during all the experimental period, it couldn't be detected the differences between the application of the $\mathrm{SO}_{2}$ and the use of the branching. The lower index of microbiological contamination and the preference of the judges in the sensorial panel had been attributed to the submitted treatments to the branching, addition of $500 \mathrm{~mL} . \mathrm{L}^{-1}$ of sodium benzoate and addition of $200 \mathrm{~mL} . \mathrm{L}^{-1}$ of sulfite dioxide.
\end{abstract}

Index-Terms: Mangifera indica L., agro-industry, physics properties, sensory evaluation.

\section{INTRODUÇ̃̃̃O}

Com uma produção de aproximadamente 850 mil t de manga(FAO, 2006), o Brasil ocupa a $5^{\mathrm{a}}$ posição entre os maiores produtores mundiais desse fruto. Recentemente, contratos fechados com o mercado nipônico têm incentivado a expansão das áreas de cultivo, não só no Vale do São Francisco, sul da Bahia ou no interior tropical de São Paulo, mas também em regiões da Amazônia Setentrional, como é o caso do Estado de Roraima, considerado por muitos como o celeiro produtivo Amazônico. Vale ressaltar que os pomares atuais, tradicionais e regionais de mangas Bourbon, Espada, Coqueiro e Ouro, estão sendo gradativamente substituídos, por pressão natural do mercado externo, pelas variedades Tommy Atkins, Haden, Keitt $e$ Palmer (Lizada, 1993).

No entanto, muitos são os empecilhos à sua plena

\footnotetext{
(Trabalho 123-06). Recebido em : 21-08-2006. Aceito para publicação em : 02-07-2007

${ }^{2}$ UFRR - Prof.. Dep ${ }^{\text {to }}$ de Fitotecnia - Centro de Ciências Agrárias, Km 12 BR174 s/nº, CEP 69301-970, Boa Vista-RR. rapelbtu@hotmail.com

${ }^{3}$ UFRR - Graduando em Agronomia - Centro de Ciências Agrárias, Km 12 BR174 s/nº, CEP 69301-970, Boa Vista-RR. rmbenedette@hotmail.com

${ }^{4}$ UFRR - Pós-graduando em Ciência e Tecnologia de Alimentos - Centro de Ciências Agrárias, Km 12 BR174 s/nº, CEP 69301-970, Boa Vista-RR.

e8campos@bol.com.br,marcosprill@bol.com.br

${ }^{5}$ UNESP/FCA - Prof. Dep ${ }^{\text {to }}$ de Gestão e Tecnologia Agroindustrial, C.P. 237, CEP 18603-970, Botucatu-SP. vieites@fca.unesp.br
} 
estabilização como pacote rentável e comercial aos produtores: a alta perecibilidade e suscetibilidade a doenças (Lizada, 1993); a elevada produção em uma sazonalidade curta e coincidente com frutos, como o pêssego e abacaxi, gerando assim forte concorrência e a conseqüente queda dos preços (Pelegrine, 1999); problemas logísticos de embalagem no acondicionamento póscolheita e no armazenamento refrigerado, péssimas condições das vias de escoamento rurais, vicinais e até mesmo principais, além da não-manutenção da cadeia do frio durante o transporte ao mercado atacadista. Esses fatores, segundo Leite (1998), condicionam a perdas anuais estimadas em até $20 \%$, por exemplo, no mercado paulista.

Neste sentido, frente ao cenário problemático da superprodução e dos elevados índices de perdas pós-colheita, a agroindustrialização de mangas pode constituir uma alternativa viável ao produtor brasileiro, regulando o preço do mercado do fruto in natura e, supostamente, aproveitando os excedentes que seriam descartados. Segundo Gonçalves (2000), além de uma pauta mais do que viável à exportação, o processamento de frutos na forma de polpas, sucos concentrados e/ou purês, constitui um produto final elaborado e, conseqüentemente, pode agregar valor nas etapas de comercialização.

Entretanto, a manutenção dos atributos qualitativos sensoriais e, sobretudo, da segurança microbiológica, pode funcionar como um condicionante básico e restritivo à abertura deste mercado diferenciado.

Rajashekhara et al. (2000), estudando a eficiência de tratamentos térmicos e conservantes na inativação de ascósporos de Neosartoria fisheri em suco de manga e uva, relataram o efeito positivo da pasteurização, somatizado, por sua vez, ao incremento do sorbato de potássio e/ou do benzoato de sódio. Baixos teores de pH $(3,5$ a 4,0) também constituem importante ferramenta no controle microbiológico (Sugai, 2002). Nesse sentido, a acidificação do produto processado, principalmente através do ácido cítrico, possibilita o controle microbiológico sem a necessidade de tratamentos térmicos agressivos (Sugai, 2002).

Considerando a dificuldade em comercializar produtos congelados nas regiões com menor poder aquisitivo, este trabalho objetivou-se na viabilização de uma tecnologia alternativa ao congelamento das polpas de frutos, avaliando, por sua vez, a eficiência da pasteurização e de preservativos em polpas de manga Tommy Atkins refrigeradas.

\section{MATERIAL E MÉTODOS}

O presente trabalho foi desenvolvido no Laboratório de Fitotecnia da Universidade Federal de Roraima (UFRR). Os frutos foram colhidos fisiologicamente maturos na propriedade agrícola do Sr. Airton Soligo, situada no município de Boa Vista-RR (Lat. $2^{\circ} 50^{\prime} 06^{\prime \prime} \mathrm{N}$ e Long. $\left.60^{\circ} 40^{\prime} 28^{\prime \prime} \mathrm{W}\right)$. Antes do ajuste de $\mathrm{pH}$ e da atividade de água, as polpas de manga apresentavam sólidos solúveis (SS) médios de 9,2 ${ }^{\circ}$ Brix, acidez titulável (AT) média de $1,13 \mathrm{~g}$ de ácido cítrico, $100 \mathrm{~g}$ de polpa ${ }^{-1}, \mathrm{pH}$ médio de 3,45 e atividade de água média de 0,98 . Esses dados comprovam que as polpas estavam dentro dos padrões de qualidade exigidos pelo
Ministério da Agricultura, Pecuária e Abastecimento (2000). Antes da confecção dos tratamentos, os frutos foram higienizados em solução de hipoclorito de sódio $(\mathrm{NaOCl})$ a $100 \mathrm{mg}$. $\mathrm{L}^{-1}$, por 10 minutos. Após os frutos serem triturados em um mixer, foi realizado o ajuste do $\mathrm{pH}$ das polpas para 3,0, através da adição de $1,88 \mathrm{~g}$ de ácido cítrico. $100 \mathrm{~g}^{-1}$ de polpa, e o ajuste da $\mathrm{A}_{\mathrm{a}}$ para 0,95 , através da adição de $39,5 \%$ de sacarose. As variáveis utilizadas foram a pasteurização (água fervente a $95 \pm 5^{\circ} \mathrm{C}$, por 0 e 1 minuto), a adição de benzoato de sódio nas concentrações de $0 ; 200$ e 500 mL.L $L^{-1}$, e dióxido de enxofre $\left(\mathrm{SO}_{2}\right)$ a $0 ; 100$ e $200 \mathrm{~mL} . \mathrm{L}^{-1}$. Os tratamentos, compostos pelas combinações entre as variáveis citadas acima, foram então embalados em sacos de PEBD de $0,060 \mathrm{~mm}(0,15 \times 0,17 \mathrm{~m}$ - sem espaço livre, termosseladas e contendo $500 \mathrm{~g}$ de polpa), e armazenadas em câmara frigorífica a $20 \pm 1{ }^{\circ} \mathrm{C}$ e $80 \pm 3 \%$ de U.R, por 28 dias. Essas condições de temperatura e umidade durante a frigoconservação das polpas foram pré-ajustadas, visando ao estabelecimento de um perfil comparativo bem próximo à realidade comercial detectada em alguns hipermercados localizados em Belém-PA, Boa Vista-RR e Manaus-AM. Durante esse período, foram analisados nos dias $1 ; 4 ; 8 ; 12 ; 16 ; 20 ; 24$ e 28 de armazenamento refrigerado (AR), sempre depois de 8 horas da saída da câmara frigorífica: os sólidos solúveis (SS), determinados através da leitura refratométrica direta, com o refratômetro tipo Abbe, marca ATAGO - N1, resultados expressos em ${ }^{\circ}$ Brix; a acidez total (AT), determinada através de titulometria de neutralização $(\mathrm{NaOH}=0,01 \mathrm{~N})$, com ponto de viragem no $\mathrm{pH}=8,2$ e resultados expressos em $\mathrm{g}$ de ácido cítrico. $100 \mathrm{~g}$ de polpa $^{-1} ; \mathrm{o} \mathrm{pH}$, determinado através de potenciometria em amostra triturada e homogeneizada; o teor de ácido ascóbico (mg por $100 \mathrm{~g}$ de polpa), determinado segundo método de Carvalho et al. (1990), que se baseia na redução do indicador 2,6 - diclorobenzenoindolfenol (DCFI) pelo ácido ascórbico; o desenvolvimento de mofos, leveduras, e coliformes fecais e totais, segundo metodologia proposta por American Public Health Association (2001) e citado por Costa et al. (2003), obedecendo inclusive à Resolução RCD $\mathrm{n}^{\circ} 12$, de 2 de janeiro de 2001, da Agência Nacional de Vigilância Sanitária (ANVISA). Realizou-se, ao final do experimento, um teste de preferência (suco feito com os tratamentos), contando com quinze julgadores treinados, através de escala hedônica de 5 pontos (Moraes, 1988) - gostei muitíssimo (nota 5); gostei muito (nota 4); gostei (nota 3); desgostei muito (nota 2), e desgostei muitíssimo (nota 1).

O delineamento experimental empregado foi o inteiramente casualizado, seguindo esquema fatorial 18x4 (18 tratamentos e 4 períodos de armazenamento refrigerado), com 5 repetições, contendo 5 amostras (embalagens) cada repetição. Os dados foram submetidos à análise de variância (teste F), e comparação de médias foi efetuada pelo teste de Tukey, a 5\%.

\section{RESULTADOS E DISCUSSÃO}

\section{Características microbiológicas}

Em referência à qualidade higiênico-sanitária das polpas de manga, em todos os tratamentos testados, em todo o período experimental, os níveis de Salmonella e de coliformes totais/ 
fecais encontravam-se dentro dos padrões microbiológicos estabelecidos pela Anvisa (2001) (Tabela 1). Em estudo similar, Costa et al. (2003), trabalhando com polpas de cupuaçu, ressaltaram a ação positiva dos mesmos obstáculos tecnológicos sugeridos neste trabalho, quanto à adequada manutenção da qualidade microbiológica, principalmente através do efetivo controle dos microrganismos patogênicos. Pereira et al. (2003), trabalhando com goiabas variedade Paloma, e Bonnas et al. (2003), trabalhando com abacaxis cv. Smooth Cayenne, também descreveram a importância e a eficiência da sanificação com o $\mathrm{NaOCl}$ na contribuição para o controle de tais contaminantes. Ainda, segundo Bonnas et al. (2003), a presença de coliformes pode funcionar como excelente parâmetro das condições de higiene aplicadas durante o processamento. Nesse sentido, baseado nos resultados aqui apresentados, podemos concluir que a produção das polpas de manga neste experimento seguiu adequadamente o controle higiênico-sanitário, não submetendo as mesmas a riscos microbiológicos indesejáveis.

Entretanto, mesmo com o controle adequado dos contaminantes patogênicos, a exemplo do que ocorreu com o trabalho de Costa et al. (2003), verificou-se constante desenvolvimento de mofos e leveduras, principalmente nos tratamentos não-submetidos ao benzoato de sódio e à pasteurização (Tabela 2). Desse modo, enquanto nas polpas simplesmente submetidas à pasteurização, a presença de mofos e leveduras não ultrapassou valores médios de $6,67.10^{4} \mathrm{UFC} \mathrm{g}^{-1}$ aos 28 dias de armazenamento, as polpas não-pasteurizadas e sem a adição do benzoato de sódio apresentavam valores médios entre $3,34.10^{6}$ a $1,14.10^{6} \mathrm{UFC} . \mathrm{g}^{-1}$, ou seja, mesmo que em que níveis ainda não-satisfatórios, o simples emprego da pasteurização diminuiu quase que pela metade a ação microbiana decompositora. Em estudo realizado por Carvalho Filho \& Massaguer (1997), trabalhando com mangas processadas na forma de purê, pôde-se novamente constatar a eficácia do tratamento térmico na contenção do desenvolvimento microbiano. No presente trabalho, os efeitos tecnológicos da pasteurização em relação à diminuição da ação de mofos e leveduras foram potencializados pela utilização do benzoato de sódio. Sendo assim, o melhor tratamento, polpa pasteurizada e tratada com $\mathrm{SO}_{2}$ a $200 \mathrm{~mL} . \mathrm{L}^{-1}$ e benzoato de sódio a $500 \mathrm{~mL} . \mathrm{L}^{-1}$, apresentou valores médios de $1,14.10^{2} \mathrm{UFC} \cdot \mathrm{g}^{-1}$. No entanto, vale ressaltar que as polpas pasteurizadas e submetidas ao benzoato de sódio na concentração de $200 \mathrm{~mL}$. $\mathrm{L}^{-1}$, com ou sem a adição do $\mathrm{SO}_{2}$, também apresentaram médias satisfatórias e próximas à citada anteriormente, apresentando valores médios entre $8,80.10^{2} \mathrm{a}$ $5,27.10^{2}$ UFC.g ${ }^{-1}$. Da mesma forma, comprovando a ação do benzoato de sódio também nas polpas não-pasteurizadas, independentemente da adição do $\mathrm{SO}_{2}$, foram detectadas médias não-superiores a $7,55 \cdot 10^{3} \mathrm{UFC} \cdot \mathrm{g}^{-1}$. Esses resultados, evidentemente, não contemplam a qualidade microbiológica aqui requerida e proposta, mas, por outro lado, também não deixam dúvidas quanto a ação antimicrobiana do benzoato de sódio. Sugere-se então, através dos resultados obtidos, que tanto a pasteurização quanto o uso do benzoato de sódio, separadamente, ou especialmente consorciados, podem reduzir a carga de mofos e leveduras em polpas de manga não- congeladas. Pina et al. (2003), utilizando-se de processos térmicos e preservativos combinados em mangas cv. Caité, semelhantemente aos empregados neste experimento, também obtiveram a estabilidade microbiológica satisfatória do produto final. O mesmo foi verificado por Costa et al. (2003), trabalhando com polpas de cupuaçu resfriadas, onde, com benzoato de sódio, em todas as concentrações empregadas, foram observadas contagens de bolores e leveduras entre $1,5.10^{1} \mathrm{UFC}^{-g^{-1} \mathrm{e}} 7,5.10^{1}$ $\mathrm{UFC} . \mathrm{g}^{-1}$, após sete dias de armazenagem à temperatura ambiente. No mesmo experimento, a polpa sem nenhum tratamento apresentou números de $2,3.10^{8} \mathrm{UFC}_{\mathrm{g}^{-1}}$ para bolores e leveduras, para o mesmo período.

Quanto à ação do $\mathrm{SO}_{2}$ nos tratamentos acima descritos, ou seja, em polpas pasteurizadas ou não, tratadas ou não com o benzoato de sódio, não se constatou diferença significativa entre os tratamentos testados ao final do experimento, principalmente em relação ao escurecimento enzimático das polpas. Mesmo porque, essa fisiopatia não fora constatada em nenhum momento durante os 28 dias de avaliação. Dados esses concordantes com Severini et al. (2001), em trabalho realizado com batatas, onde a pasteurização por si só se mostrou capaz na prevenção do escurecimento enzimático. No entanto, Haminiuk et al. (2005), trabalhando com maçãs Fuji e Gala, observaram que, apesar de o pré-tratamento imersão em água fervente ter apresentando maior relevância na prevenção do escurecimento, os frutos submetidos ao pré-tratamento imersão em solução de $\mathrm{SO}_{2}$ a $0,25 \%$ alcançaram maior aceitação no painel sensorial. Dados esses discordantes dos obtidos neste experimento, onde a ausência do $\mathrm{SO}_{2}$ não foi fator condicionante ao surgimento ou não do escurecimento enzimático nas polpas, nem tampouco influenciou na decisão final dos julgadores (Figura 2). Sendo assim, dada a ausência de escurecimento em todos os tratamentos, os autores são incapazes de estabelecer quem foi o responsável direto pela contenção dessa fisiopatia nas polpas de manga.

\section{Características sensoriais}

A pasteurização e os demais preservativos químicos utilizados no experimento interagiram diferentemente no desenvolvimento dos atributos qualitativos das polpas de manga. Nesse sentido, observa-se que somente nas análises de SS e AT é que os tratamentos não se diferenciaram entre si (Tabela 3). Antes do ajuste da $\mathrm{A}_{\mathrm{a}}$ e do $\mathrm{pH}$, as polpas apresentavam, em média, $10,2^{\circ}$ Brix e $1,17 \%$ de ácido cítrico. $100 \mathrm{~g}$ de polpa $^{-1}$. Esses resultados mostram que as polpas estavam dentro dos padrões de identidade e qualidade estabelecidos pelo Ministério da Agricultura e do Abastecimento (2000). Depois do ajuste, as polpas passaram a apresentar teores de SS variando entre 47,3 e $45,8^{\circ}$ Brix, e níveis de AT entre 2,24 e 2,31\% de ácido cítrico. $100 \mathrm{~g}$ de polpa ${ }^{-1}$. Esse comportamento indica que não houve influência significativa dos tratamentos combinados no metabolismo de transformação dos ácidos orgânicos e dos açúcares solúveis em água.

Ainda sobre os parâmetros físico-químicos das polpas de manga, pôde-se estabelecer um paralelo comparativo entre os resultados microbiológicos (Tabela 2), com as variações de $\mathrm{pH}$ observadas nos tratamentos ao longo do período experimental 
(Figura 1). Autores como Guerrero et al. (1994) e López-Malo et al. (1994) sugerem teores de $\mathrm{pH}$ na faixa de 3,1 a 3,5, para elaboração de polpas de frutos. Nesse sentido, somente os tratamentos pasteurizados e tratados com o benzoato de sódio na concentração de $500 \mathrm{~mL} . \mathrm{L}^{-1}$, com teores entre 3,47 e 3,29, estavam enquadrados dentro da faixa de $\mathrm{pH}$ supracitada. Coincidentemente, a atividade de mofos e leveduras nesses tratamentos esteve bem controlada (Tabela 2). Comprova-se então a afirmação de Sugai (2002), de que a manutenção dos teores de $\mathrm{pH}$, em teores compreendidos entre 3,5 e 4, pode constituir importante ferramenta no controle microbiológico. Já nas polpas não-pasteurizadas e não-submetidas à ação do benzoato de sódio, onde se detectou o maior desenvolvimento de mofos e leveduras, o $\mathrm{pH}$ apresentou-se relativamente elevado. Nesses tratamentos, o pH variou de 3,00 após a adequação da $\mathrm{A}_{\mathrm{a}} \mathrm{e}$ do $\mathrm{pH}$, para teores entre 3,98 e 4,06 aos sete dias de experimentação, chegando ao final dos 28 dias de armazenamento com teores próximos a 6,05 . Portanto, partindo-se do pressuposto de que a manutenção do pH em níveis mais baixos consista em um importante fator de restrição ao crescimento de microrganismos nos alimentos, o elevado $\mathrm{pH}$ observado nesses tratamentos pode ter influência direta nos elevados índices de contaminação microbiológica dessas polpas ou, até mesmo, ser um reflexo da intensa atividade microbiana.

Quanto ao teor de ácido ascórbico nas polpas de mangas, os maiores valores, contrariamente aos demais resultados aqui comentados e discutidos, foram detectados nas polpas nãopasteurizadas. Explicando o fato, segundo Pina et al. (2003), a estabilidade da vitamina $\mathrm{C}$ nos alimentos é afetada por vários fatores, como, por exemplo, a temperatura, a luz, o oxigênio e o $\mathrm{pH}$. Esses fatores, segundo os próprios autores, principalmente quanto ao tratamento térmico nas polpas pasteurizadas, contribuíram definitivamente para a diminuição acentuada das concentrações de ácido ascórbico nas polpas de manga. Assim sendo, todos os tratamentos submetidos à pasteurização chegaram ao final do experimento sem qualquer traço dessa vitamina em seu conteúdo. Pina et al. (2003), em trabalho semelhante com mangas cv. Caité, também verificaram que os teores de ácido ascórbico apresentaram perdas crescentes no decorrer da vida de prateleira, sendo que, já no $3^{\circ}$ mês de armazenagem, a perda de vitamina $C$ foi total. Em contraposição, os tratamentos não-pasteurizados, que nas análises microbiológicas e de $\mathrm{pH}$ obtiveram os piores desempenhos, apresentavam-se com valores de até $7,5 \mathrm{mg}$ de ácido ascórbico. $100 \mathrm{~g}$ de polpa ${ }^{-1}$, como é o caso das polpas nãopasteurizadas, porém submetidas à ação do benzoato de sódio na concentração de $500 \mathrm{~mL} . \mathrm{L}^{-1}$. Vale ressaltar novamente a ação do benzoato de sódio, agora sobre a manutenção das concentrações de ácido ascórbico nas polpas de manga.

$\mathrm{O}$ teste de preferência, englobando de uma maneira geral os atributos visuais, o odor, o sabor e o aroma dos frutos, confirmou a mesma tendência detectada nos testes laboratoriais. Nesse sentido, as polpas pasteurizadas e tratadas com o benzoato de sódio na concentração de $500 \mathrm{~mL} . \mathrm{L}^{-1}$, independentemente da adição ou não do $\mathrm{SO}_{2}$, foram as preferidas em relação aos demais tratamentos. Esse tratamento obteve unanimidade de escolha pelos julgadores. Isso nada mais é do que a conseqüência da manutenção dos atributos sensoriais verificada durante os 28 dias de armazenamento. A drástica redução da aceitação dos julgadores em relação aos tratamentos não-pasteurizados e nãosubmetidos ao benzoato de sódio, deve-se, principalmente, ao crescimento e desenvolvimento microbiano, proporcionando, por sua vez, alteração considerável na qualidade final do produto.

FIGURA 1 - (A) Ácido ascórbico e (B) pH em polpas de mangas cv. Tommy Atkins, refrigeradas e armazenadas por 28 dias a $20 \pm 1{ }^{\circ} \mathrm{C}$ e $80 \pm 3 \%$ de U.R., Boa Vista-RR - 2005 .

(A)

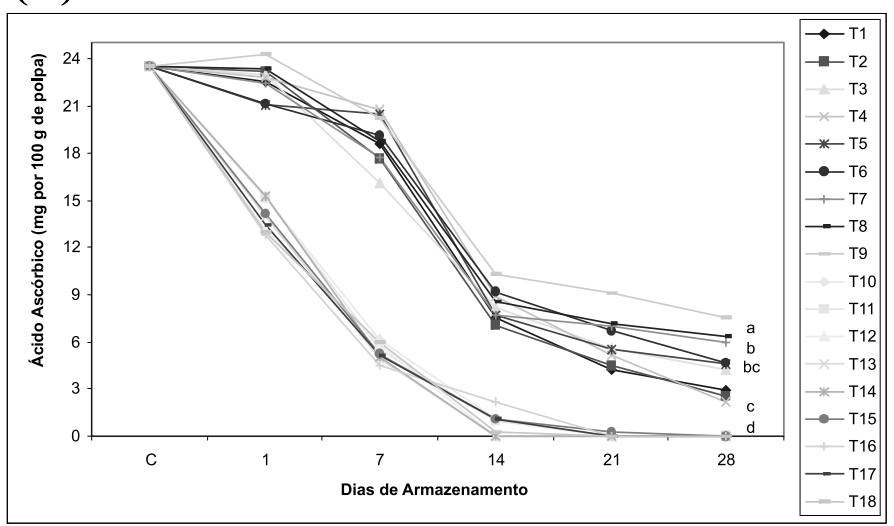

(B)

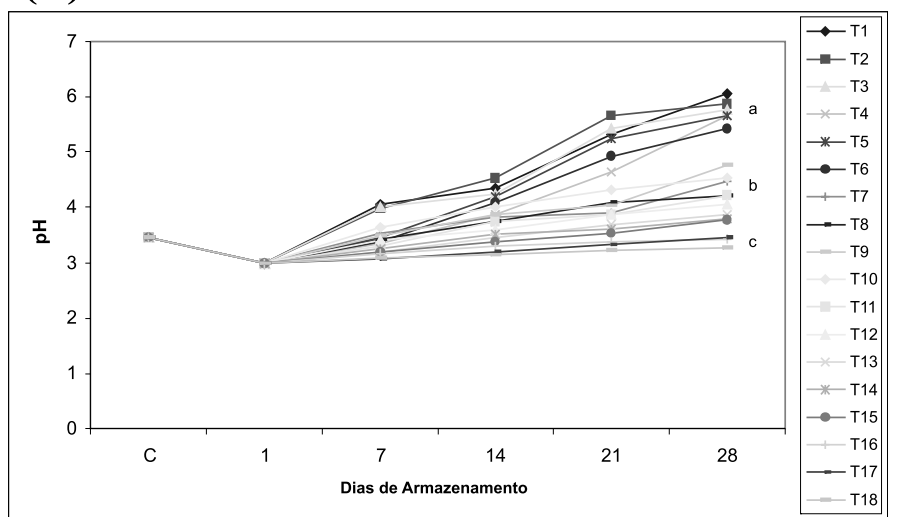

Tratamentos: T1 - controle; T2 - sem branq + 100mL.L-1 de SO; T3 - sem branq + 200mL.L-1 de SO; T4 - sem branq + $200 \mathrm{~mL} . \mathrm{L}-1$ de benzoato de sódio; T5 - sem branq $+200 \mathrm{~mL} . \mathrm{L}-1$ de benzoato de sódio $+100 \mathrm{~mL} . \mathrm{L}-1$ de $\mathrm{SO}_{2}$; T6 - sem branq $+200 \mathrm{~mL}$.L-1 de benzoato de sódio $+200 \mathrm{~mL} . \mathrm{L}-1$ de SO ;

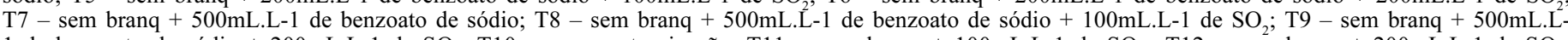
1 de benzoato de sódio $+200 \mathrm{~mL} . \mathrm{L}-1$ de $\mathrm{SO}_{2} ; \mathrm{T} 10-$ com pasteurização; T11 - com branq $+100 \mathrm{~mL} . \mathrm{L}-1$ de $\mathrm{SO}_{2}$; T12 - com branq $+200 \mathrm{~mL}$.L-1 de $\mathrm{SO}_{2}$; T13 - com branq + 200mL.L-1 de benzoato de sódio; T14 - com branq + 200mL.L-1 de benzoato de sódio + $100 \mathrm{~mL}$.L-1 de SO ; T15 - com branq + 200mL.L-1 de benzoato de sódio + 200mL.L-1 de $\mathrm{SO}_{2}$; T16 - com branq + 500mL.L-1 de benzoato de sódio; T17 - com branq + 500mL.L-1 de benzoato de sódio $+100 \mathrm{~mL}$.L-1 de $\mathrm{SO}_{2}$; T18 - com branq $+500 \mathrm{~mL} . \mathrm{L}-1$ de benzoato de sódio $+200 \mathrm{~mL} . \mathrm{L}-1$ de $\mathrm{SO}_{2}$;

A unidade utilizada para quantificar as concentrações de benzoato de sódio e $\mathrm{SO}_{2}$ é mL.L-1 (parte por milhão)

As médias seguidas das mesmas letras não diferem entre si, ao nível de $5 \%$ de probabilidade, pelo teste de Tukey. 
FIGURA 2 - Teste de preferência (escala hedônica: 1-desgostei muitíssimo a 5-gostei muitíssimo) em polpas de mangas cultivar Tommy Atkins, não-congeladas e frigoarmazenadas por 28 dias a $20 \pm 1{ }^{\circ} \mathrm{C}$ e $80 \pm 3 \%$ de U.R., Boa Vista-RR -2005.

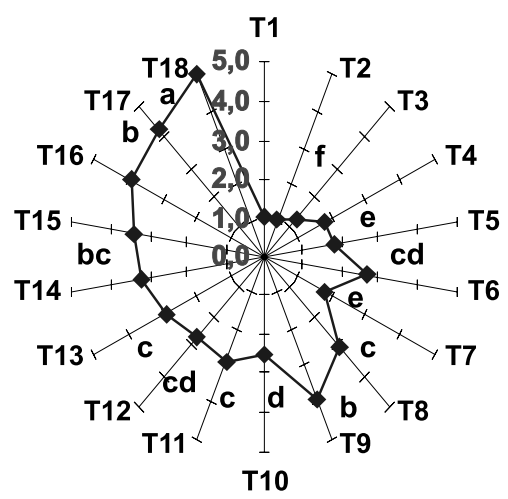

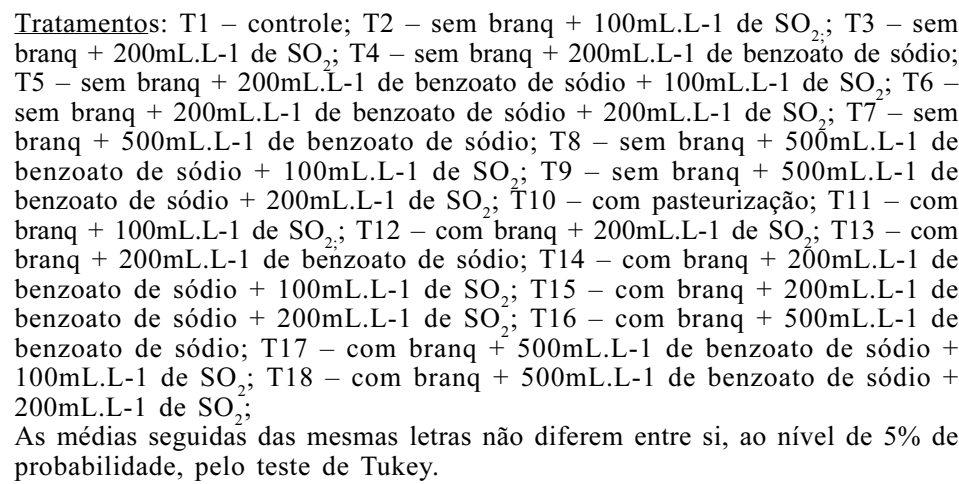

TABELA 1 - Contagem de coliformes totais e fecais, em polpas de mangas cv. Tommy Atkins, refrigeradas e armazenadas por 28 dias a $20 \pm 1{ }^{\circ} \mathrm{C}$ e $80 \pm 3 \%$ de U.R., Boa Vista-RR -2005 .

\begin{tabular}{|c|c|c|c|c|c|c|c|c|c|c|c|c|c|c|c|c|c|c|}
\hline \multirow{3}{*}{ DIAS } & \multicolumn{9}{|c|}{ Sem pasteurização } & \multicolumn{9}{|c|}{ Pasteurização por 1 minuto } \\
\hline & \multicolumn{3}{|c|}{$\begin{array}{c}\text { s/ Benzoato de } \\
\text { Potássio }\end{array}$} & \multicolumn{3}{|c|}{$\begin{array}{c}\text { Benzoato de } \\
\text { Potássio (200) }\end{array}$} & \multicolumn{3}{|c|}{$\begin{array}{c}\text { Benzoato de } \\
\text { Potássio }(500)\end{array}$} & \multicolumn{3}{|c|}{$\begin{array}{c}\text { s/ Benzoato de } \\
\text { Potássio }\end{array}$} & \multicolumn{3}{|c|}{$\begin{array}{c}\text { Benzoato de } \\
\text { Potássio (200) }\end{array}$} & \multicolumn{3}{|c|}{$\begin{array}{c}\text { Benzoato de } \\
\text { Potássio }(500)\end{array}$} \\
\hline & $\begin{array}{c}\mathrm{s} / \\
\mathrm{SO}_{2} \\
\end{array}$ & $\begin{array}{l}\mathrm{SO}_{2} \\
100 \\
\end{array}$ & $\begin{array}{l}\mathrm{SO}_{2} \\
200 \\
\end{array}$ & $\begin{array}{c}\mathrm{s} / \\
\mathrm{SO}_{2} \\
\end{array}$ & \begin{tabular}{|l|}
$\mathrm{SO}_{2}$ \\
100 \\
\end{tabular} & $\begin{array}{l}\mathrm{SO}_{2} \\
200 \\
\end{array}$ & $\begin{array}{c}\mathrm{s} / \\
\mathrm{SO}_{2} \\
\end{array}$ & $\begin{array}{l}\mathrm{SO}_{2} \\
100 \\
\end{array}$ & \begin{tabular}{|l|}
$\mathrm{SO}_{2}$ \\
200 \\
\end{tabular} & $\begin{array}{c}\mathrm{s} / \\
\mathrm{SO}_{2} \\
\end{array}$ & \begin{tabular}{|l|}
$\mathrm{SO}_{2}$ \\
100 \\
\end{tabular} & \begin{tabular}{|l|}
$\mathrm{SO}_{2}$ \\
200 \\
\end{tabular} & $\begin{array}{c}\mathrm{S} / \\
\mathrm{SO}_{2} \\
\end{array}$ & $\begin{array}{l}\mathrm{SO}_{2} \\
100 \\
\end{array}$ & \begin{tabular}{l|}
$\mathrm{SO}_{2}$ \\
200 \\
\end{tabular} & $\begin{array}{c}\mathrm{s} / \\
\mathrm{SO}_{2} \\
\end{array}$ & $\begin{array}{l}\mathrm{SO}_{2} \\
100 \\
\end{array}$ & $\begin{array}{l}\mathrm{SO}_{2} \\
200 \\
\end{array}$ \\
\hline antes ajuste & $>3 *$ & $>3$ & $>3$ & $>3$ & $>3$ & $>3$ & $>3$ & $>3$ & $>3$ & $>3$ & $>3$ & $>3$ & $>3$ & $>3$ & $>3$ & $>3$ & $>3$ & $>3$ \\
\hline 1 & $>3$ & $>3$ & $>3$ & $>3$ & $>3$ & $>3$ & $>3$ & $>3$ & $>3$ & $>3$ & $>3$ & $>3$ & $>3$ & $>3$ & $>3$ & $>3$ & $>3$ & $>3$ \\
\hline 28 & $>3$ & $>3$ & $>3$ & $>3$ & $>3$ & $>3$ & $>3$ & $>3$ & $>3$ & $>3$ & $>3$ & $>3$ & $>3$ & $>3$ & $>3$ & $>3$ & $>3$ & $>3$ \\
\hline
\end{tabular}

*Número mais provável por grama de amostra (NMP.g $\left.{ }^{-1}\right)$.

A unidade utilizada para quantificar as concentrações de benzoato de sódio e $\mathrm{SO}_{2}$ é mL.L-1

TABELA 2 - Contagem de mofos e leveduras, em polpas de mangas cv. Tommy Atkins, refrigeradas e armazenadas por 28 dias a $20 \pm$ $1{ }^{\circ} \mathrm{C}$ e $80 \pm 3 \%$ de U.R., Boa Vista-RR -2005 .

\begin{tabular}{|c|c|c|c|c|c|c|c|c|c|}
\hline \multirow{3}{*}{ DIAS } & \multicolumn{9}{|c|}{ s/ pasteurização } \\
\hline & \multicolumn{3}{|c|}{ s/ Benzoato de Potássio } & \multicolumn{3}{|c|}{ Benzoato de Potássio (200) } & \multicolumn{3}{|c|}{ Benzoato de Potássio (500) } \\
\hline & $\mathrm{s} / \mathrm{SO}_{2}$ & $\mathrm{SO}_{2} 100$ & $\mathrm{SO}_{2} 200$ & $\mathrm{~s} / \mathrm{SO}_{2}$ & $\mathrm{SO}_{2} 100$ & $\mathrm{SO}_{2} 200$ & $\mathrm{~s} / \mathrm{SO}_{2}$ & $\mathrm{SO}_{2} 100$ & $\mathrm{SO}_{2} 200$ \\
\hline $\begin{array}{l}\text { antes } \\
\text { ajuste }\end{array}$ & $2,1.10^{2} \mathrm{Ea}$ & $2,1.10^{2} \mathrm{Ea}$ & $2,1.10^{2} \mathrm{Ea}$ & $2,1.10^{2} \mathrm{Ba}$ & $2,1.10^{2} \mathrm{Ba}$ & $2,1.1$ & $0^{2} \mathrm{Ba}$ & $1.10^{2} \mathrm{Ba}$ & $2,1 \cdot 10^{2} \mathrm{Ba}$ \\
\hline 1 & $2,3.10^{1} \mathrm{Fa}$ & $1,9.10^{1} \mathrm{Fa}$ & $1,3 \cdot 10^{1} \mathrm{Fa}$ & $2,5 \cdot 10^{1} \mathrm{Ca}$ & $1,8.10^{1} \mathrm{Ca}$ & $1,2.10^{1} \mathrm{Ca}$ & $3,1.10^{1} \mathrm{Ca}$ & $2,1.10^{1} \mathrm{Ca}$ & $1,0.10^{1} \mathrm{Ca}$ \\
\hline 7 & $8,2 \cdot 10^{3} \mathrm{Da}$ & $5,3.10^{3} \mathrm{Da}$ & $1,5.10^{3} \mathrm{Da}$ & $8,1.10^{1} \mathrm{Cc}$ & $5,7.10^{1} \mathrm{Cc}$ & $7,2.10^{1} \mathrm{Cc}$ & $8,4.10^{1} \mathrm{Cc}$ & $6,9 \cdot 10^{1} \mathrm{Cc}$ & $7,5.10^{1} \mathrm{Cc}$ \\
\hline 14 & $2,4.10^{4} \mathrm{Ca}$ & $4,3 \cdot 10^{4} \mathrm{Ca}$ & $2,6.10^{4} \mathrm{Ca}$ & $7,4.10^{2} \mathrm{Bc}$ & $4,8.10^{2} \mathrm{Bb}$ & $3,1.10^{1} \mathrm{Cd}$ & $6,5.10^{2} \mathrm{Bb}$ & $4,3 \cdot 10^{2} \mathrm{Bc}$ & $5,0.10^{1} \mathrm{Cd}$ \\
\hline 21 & $5,9.10^{5} \mathrm{Ba}$ & $3,2.10^{5} \mathrm{Ba}$ & $6,1 \cdot 10^{5} \mathrm{Ba}$ & $1,3 \cdot 10^{3} \mathrm{Ac}$ & $3,4 \cdot 10^{3} \mathrm{Ac}$ & $6,1 \cdot 10^{2} \mathrm{Bd}$ & $9,3 \cdot 10^{2} \mathrm{Bd}$ & $8,2.10^{2} \mathrm{Bd}$ & $4,1 \cdot 10^{2} \mathrm{Bd}$ \\
\hline 28 & $3,3.10^{6} \mathrm{Aa}$ & $2,4.10^{6} \mathrm{Aa}$ & $1,4 \cdot 10^{6} \mathrm{Aa}$ & $3,5.10^{3} \mathrm{Ab}$ & $7,5.10^{3} \mathrm{Ab}$ & $4,1.10^{3} \mathrm{Ab}$ & $4,4.10^{3} \mathrm{Ab}$ & $6,3 \cdot 10^{3} \mathrm{Ab}$ & $4,9 \cdot 10^{3} \mathrm{Ab}$ \\
\hline DIAS & \multicolumn{9}{|c|}{ Com pasteurização } \\
\hline $\begin{array}{l}\text { antes } \\
\text { ajuste }\end{array}$ & $2,1.10^{2} \mathrm{Ca}$ & $2,1.10^{2} \mathrm{Ba}$ & $2,1.10^{2} \mathrm{Ca}$ & $2,1.10^{2} \mathrm{Aa}$ & $2,1.10^{2} \mathrm{Aa}$ & $0^{2} \mathrm{Aa}$ & $2,1.10^{2} \mathrm{Aa}$ & ${ }^{2} \mathrm{Ba}$ & $2,1.10^{2} \mathrm{Aa}$ \\
\hline 1 & $3,1.10^{1} \mathrm{Da}$ & $2,2.10^{1} \mathrm{Ca}$ & $3,3 \cdot 10^{1} \mathrm{Da}$ & $3.7 .10^{1} \mathrm{Ba}$ & $2,3 \cdot 10^{1} \mathrm{Ba}$ & $3,19 \cdot 10^{1} \mathrm{Ba}$ & $2,1.10^{1} \mathrm{Ba}$ & $3,9.10^{1} \mathrm{Ca}$ & $2,2.10^{1} \mathrm{Ba}$ \\
\hline 7 & $9,1.10^{2} \mathrm{Cb}$ & $2,1 \cdot 10^{3} \mathrm{Ba}$ & $7,2 \cdot 10^{2} \mathrm{Cb}$ & $5.5 .10^{1} \mathrm{Bc}$ & $5,9.10^{1} \mathrm{Bc}$ & $6,20 \cdot 10^{1} \mathrm{Bc}$ & $7,8 \cdot 10^{1} \mathrm{Bc}$ & $6.1 .10^{1} \mathrm{Bc}$ & $5,8 \cdot 10^{1} \mathrm{Bc}$ \\
\hline 14 & $1,1.10^{2} \mathrm{Cb}$ & $7,2.10^{3} \mathrm{Bb}$ & $3,3.10^{3} \mathrm{Bb}$ & $7,4 \cdot 10^{1} \mathrm{Bc}$ & $1,5.10^{2} \mathrm{Ac}$ & $9,27 \cdot 10^{2} \mathrm{Ac}$ & $9,9 \cdot 10^{1} \mathrm{Bc}$ & $8,2 \cdot 10^{1} \mathrm{Bc}$ & $7,2 \cdot 10^{1} \mathrm{Bc}$ \\
\hline 21 & $8,3 \cdot 10^{3} \mathrm{Ac}$ & $1,1.10^{4} \mathrm{Ab}$ & $7,9.10^{3} \mathrm{Bc}$ & $3,4.10^{2} \mathrm{Ad}$ & $4,0.10^{2} \mathrm{Ad}$ & $3,39.10^{2} \mathrm{Ad}$ & $2,5.10^{2} \mathrm{Ab}$ & $1,1.10^{2} \mathrm{Ad}$ & $9,3 \cdot 10^{1} \mathrm{Be}$ \\
\hline 28 & $6,6 \cdot 10^{4} \mathrm{Ab}$ & $4,4 \cdot 10^{4} \mathrm{Ab}$ & $1,0.10^{4} \mathrm{Ab}$ & $8,8 \cdot 10^{2} \mathrm{Ac}$ & $8,1.10^{2} \mathrm{Ac}$ & $7,75.10^{2} \mathrm{Ac}$ & $5,2.10^{2} \mathrm{Ac}$ & $6,8 \cdot 10^{2} \mathrm{Ac}$ & $1,1.10^{2} \mathrm{Ad}$ \\
\hline
\end{tabular}

*Unidades formadoras de colônia por grama de amostra (UFC. $\mathrm{g}^{-1}$ ).

A unidade utilizada para quantificar as concentrações de benzoato de sódio e $\mathrm{SO}_{2}$ é $\mathrm{mL}^{-\mathrm{L}^{-1}}$

As médias seguidas das mesmas letras, nas linhas e nas colunas, não diferem entre si, ao nível de $5 \%$ de probabilidade, pelo teste de Tukey. 
TABELA 3 - Sólidos solúveis e acidez titulável em polpas de mangas cv. Tommy Atkins, refrigeradas e armazenadas por 28 dias a 20 $\pm 1{ }^{\circ} \mathrm{C}$ e $80 \pm 3 \%$ de U.R., Boa Vista-RR -2005 .

\begin{tabular}{|c|c|c|c|c|c|c|c|c|c|c|c|c|c|c|c|c|c|c|c|}
\hline \multirow{3}{*}{ Análises } & \multirow{3}{*}{ Dias } & \multicolumn{9}{|c|}{ s/ pasteurização } & \multicolumn{9}{|c|}{ Pasteurização por 1 minuto } \\
\hline & & \multicolumn{3}{|c|}{$\begin{array}{l}\text { s/ Benzoato de } \\
\text { Potássio }\end{array}$} & \multicolumn{3}{|c|}{$\begin{array}{c}\text { Benzoato de } \\
\text { Potássio }(200)\end{array}$} & \multicolumn{3}{|c|}{$\begin{array}{c}\text { Benzoato de } \\
\text { Potássio }(500)\end{array}$} & \multicolumn{3}{|c|}{$\begin{array}{l}\text { s/ Benzoato de } \\
\text { Potássio }\end{array}$} & \multicolumn{3}{|c|}{$\begin{array}{c}\text { Benzoato de } \\
\text { Potássio }(200)\end{array}$} & \multicolumn{3}{|c|}{$\begin{array}{c}\text { Benzoato de } \\
\text { Potássio (500) }\end{array}$} \\
\hline & & \begin{tabular}{c|}
$\mathrm{s} /$ \\
$\mathrm{SO}_{2}$
\end{tabular} & $\begin{array}{l}\mathrm{SO}_{2} \\
100\end{array}$ & $\begin{array}{l}\mathrm{SO}_{2} \\
200\end{array}$ & \begin{tabular}{|c|}
$\mathrm{S} /$ \\
$\mathrm{SO}_{2}$ \\
\end{tabular} & $\begin{array}{l}\mathrm{SO}_{2} \\
100\end{array}$ & $\begin{array}{l}\mathrm{SO}_{2} \\
200\end{array}$ & \begin{tabular}{|c|}
$\mathrm{s} /$ \\
$\mathrm{SO}_{2}$ \\
\end{tabular} & $\begin{array}{l}\mathrm{SO}_{2} \\
100\end{array}$ & $\begin{array}{l}\mathrm{SO}_{2} \\
200\end{array}$ & \begin{tabular}{|c|}
$\mathrm{s} /$ \\
$\mathrm{SO}_{2}$ \\
\end{tabular} & $\begin{array}{l}\mathrm{SO}_{2} \\
100\end{array}$ & \begin{tabular}{|l|}
$\mathrm{SO}_{2}$ \\
200
\end{tabular} & \begin{tabular}{|c|}
$\mathrm{s} /$ \\
$\mathrm{SO}_{2}$ \\
\end{tabular} & \begin{tabular}{|c|}
$\mathrm{SO}_{2}$ \\
100 \\
\end{tabular} & $\begin{array}{l}\mathrm{SO}_{2} \\
200\end{array}$ & \begin{tabular}{|c|}
$\mathrm{s} /$ \\
$\mathrm{SO}_{2}$ \\
\end{tabular} & \begin{tabular}{|c|}
$\mathrm{SO}_{2}$ \\
100 \\
\end{tabular} & $\begin{array}{l}\mathrm{SO}_{2} \\
200\end{array}$ \\
\hline \multirow{3}{*}{$\mathrm{SS}^{* *}$} & $\mathrm{C}^{*}$ & 9,2 & 9,2 & 9,2 & 9,2 & 9,2 & 9,2 & 9,2 & 9,2 & 9,2 & 9,2 & 9,2 & 9,2 & 9,2 & 9,2 & 9,2 & 9,2 & 9,2 & 9,2 \\
\hline & 1 & 45,1 & 44,2 & 45,2 & 44,8 & 44,9 & 45,2 & 45,4 & 44,9 & 44,8 & 45 & 45,3 & 45,4 & 45,2 & 44,9 & 45,4 & 45,3 & 45,5 & 44,8 \\
\hline & 28 & 46,4 & 47,1 & 45.8 & 46,5 & 45,9 & 46,8 & 47,1 & 46 & 47,2 & 46,7 & 47,1 & 47,3 & 46,7 & 47,2 & 46 & 46,9 & 47,2 & 46,8 \\
\hline \multirow{3}{*}{$\mathrm{AT}^{* *}$} & $\mathrm{C}$ & 1,13 & 1,13 & 1,13 & 1,13 & 1,13 & 1,13 & 1,13 & 1,13 & 1,13 & 1,13 & 1,13 & 1,13 & 1,13 & 1,13 & 1,13 & 1,13 & 1,13 & 1,13 \\
\hline & 1 & 2,25 & 2,25 & 2,31 & 2,28 & 2,27 & 2,29 & 2,26 & 2,24 & 2,28 & 2,25 & 2,24 & 2,25 & 2,26 & 2,28 & 2,27 & 2,30 & 2,25 & 2,29 \\
\hline & 28 & 1,88 & 1,95 & 1,99 & 1,95 & 1,86 & 1,94 & 1,88 & 1,96 & 1,94 & 1,85 & 1,94 & 1,94 & 1,88 & 1,94 & 1,96 & 1,96 & 1,95 & 1,87 \\
\hline
\end{tabular}

* Dados obtidos após a colheita dos frutos.

** Não foram detectadas diferenças significativas, ao ao nível de $5 \%$ de probabilidade, pelo teste de Tukey.

A unidade utilizada para quantificar as concentrações de benzoato de sódio e $\mathrm{SO}_{2}$ é $\mathrm{mL}^{-L^{-1}}$

\section{CONCLUSÃO}

Neste trabalho, onde prioritariamente se pretenderam estabelecer diretrizes tecnológicas adaptadas à realidade do pequeno agroempresário, constatou-se que, para a conservação de polpas de manga cv. Tommy Atkins, refrigeradas e armazenadas por até 28 dias, respeitando-se os limites de segurança microbiológica e sensorial, é indicada a utilização da pasteurização por 1 minuto associada a $500 \mathrm{~mL} . \mathrm{L}^{-1}$ de benzoato de sódio e $200 \mathrm{~mL} . \mathrm{L}^{-1}$ de dióxido de enxofre. Entretanto, diante dos resultados anteriormente dispostos, sugere-se também a continuidade do estudo em relação ao dióxido de enxofre, principalmente quanto ao escurecimento enzimático.

\section{REFERÊNCIAS}

AGÊNCIA NACIONAL DE VIGILANCIA SANITÁRIA. Resolução RCD n. 12, de 2 de janeiro de 2001. Disponível em: $<$ http://www.anvisa.gov.br.2001>.

BONNAS, S.D.; CHITARRA, A.B,; PRADO, M.E.T.; JÚNIOR, D.T. Qualidade do abacaxi cv. Smooth Cayenne minimamente processado. Revista Brasileira de Fruticultura, Jaboticabal, v. 25, n.2, p. 206-209, 2003.

BRASIL. MINISTÉRIO DA AGRICULTURA E DO ABASTECIMENTO. Instrução Normativa $\mathrm{n}^{\circ} 1$ de 07 de janeiro de 2000. Aprova o Regulamento Técnico para fixação dos Padrões de Identidade e Qualidade para polpa de fruta. Diário Oficial da União, Brasília-DF,Seção I, p.54.

CARVALHO, C. R. L.; MANTOVANI, D. M. B.; CARVALHO, P. R. N.; MORAES, R. M. M. Análises químicas de alimentos: manual técnico. Campinas: Instituto de Tecnologia de Alimentos, 1990. $121 \mathrm{p}$.
CARVALHO FILHO, C.D.; MASSAGUER, P.R. Processamento térmico de purê de banana (Musa cavendishii, lamb.) em embalagens flexíveis esterilizáveis. Ciência e Tecnologia de Alimentos, Campinas, v.17, n.3, p.213-218, 1997.

COSTA, M.C; MAIA, G.A.; SOUZA FILHO, M. de S.M MEN; FIGUEIREDO, R.W. de; NASSU, R.T.; MONTEIRO, J.C.S. Conservação de polpa de cupuaçu [Theobroma grandiflorum (Willd. Ex Spreng.) Schum] por métodos combinados. Revista Brasileira de Fruticultura, Jaboticabal, v.25 n.2, p. 115-118, 2003

FAO, FAOSTAT Agriculture Data. Disponível em: < http:// faostat.fao.org > . Acesso em: 02 fev. 2006.

GONÇALVES, C.E. Exportações agroindustriais brasileiras: valor industrial x valor de mercado. São Paulo: FIPE, 2000.

HAMINIUK, C.W.I, OLIVEIRA, C.R.G, BAGGIO1, E.C.R.,MASSON, M.L. Efeito de pré-tratamentos no escurecimento das cultivares de maçã fuji e gala após o congelamento. Ciência e Agrotecnologia, Lavras, v.29, n.5, p.1029-1033., 2005.

JAGTIANI, J.; CHAN JR., H. T., SAKAI, W. S. Tropical fruit processing: food science and technology. San Diego: Academic Press, 1988. 184p.

LEITE, L.A.S. O agronegócio manga no nordeste do Brasil. In: EMBRAPA. Cadeias produtivas e sistemas naturais: prospecção tecnológica. Brasília: EMBRAPA-SPI, 1998. p.389-489.

LIZADA, C. Mango. In: SEYMOR, G.; TAYLOR, J.; TUCKER, G. Biochemistry of fruit ripening. London: Chapman \& Hall, 1993. p.255-271. 
LÓPEZ-MALO, A.; PALOU, E.; WELTI, J.; CORTE, P. e ARGAIZ, A. Shelf-stable higt moisture papaya minimally processed by combined methods. Food Research International Kidlington, v.27, p.545-553, 1994.

PELEGRINE, D.H. Comportamento reológico das polpas de manga e abacaxi. 1999. 115f. Dissertação (Mestrado em Engenharia de Alimentos) - Faculdade de Engenharia de Alimentos, Universidade Estadual de Campinas, Campinas, 1999.

PEREIRA, L.M.; RODRIGUES, A.C.C.; SARANTÓPOULOS, C.I.G.de L. Vida-de-prateileira de goiabas minimamente processadas acondicionadas em embalagens sob atmosfera modificada. Ciência e Tecnologia de Alimentos, Campinas, v. 23, n.3, p. 427-433, 2003.
SEVERINI, C.; PILLI, T. de; BAIANO, A.; MASTROCOLA, D.; MASSINI, R. Preventing enzymatic browning of potato by microwave blanching. Sciences des Aliments, Paris, v. 21, p. 149160,2001

SUGAI, A.Y. Processamento descontínuo de purê de manga (Mangifera indica Linn.), variedade Haden: estudo de viabilidade produto pronto para o consumo. 2002. $82 \mathrm{f}$. Dissertação (Mestrado em Engenharia) - Escola Politécnica, Universidade de São Paulo, São Paulo, 2002. 